\title{
Evaluación de la efectividad y satisfacción de las pacientes tras miomectomía histeroscópica en consulta
}

\section{Evaluation of effectiveness and patient satisfaction of hysteroscopic myomectomy in office}

Rocío García-Jiménez*, Irene Valero, María D. Lara, Carlota García-Salmones, Ignacio Salazar y Rosa Oña Unidad de Obstetricia y Ginecología, Hospital Universitario Nuestra Señora de Valme, Sevilla, España

\section{Resumen}

Objetivo: Analizar la efectividad de las miomectomías histeroscópicas en consulta realizadas con minirresector y conocer si hay factores relacionados con el grado de satisfacción de las pacientes. Método: Estudio observacional, transversal y prospectivo, de mujeres sometidas a miomectomía histeroscópica en consulta durante el año 2018. Las pacientes recibieron medicación para la preparación cervical, analgesia oral y anestesia paracervical. La miomectomía se realizó con un minirresector de $5.8 \mathrm{~mm}$. Se registraron el tiempo y el dolor en una escala visual analógica (EVA) durante la entrada y la resección, así como la satisfacción de las pacientes a los 3 meses con el cuestionario validado CSQ-8. Resultados: El estudio incluyó 59 pacientes. El tiempo medio de entrada fue menor de 1 minuto (47,93 segundos) y el de resección fue de 13,51 minutos. El dolor referido por las pacientes en la EVA durante la entrada y la resección puntuó en torno a 3 y 4 , respectivamente. Se consiguió un $74.6 \%$ de resecciones completas de los miomas y la puntuación media de satisfacción de las pacientes fue de 27.17. La resección completa del mioma se asoció con una mayor satisfacción total de las pacientes. Conclusiones: La miomectomía histeroscópica en consulta llevada a cabo con un minirresector de $5.8 \mathrm{~mm}$ con analgesia paracervical obtiene buenos resultados clínicos, con buena satisfacción de las pacientes. Esta última se relaciona con una resección completa del mioma, sin que influyan el tiempo necesario para su exéresis ni el dolor.

Palabras clave: Miomectomía histeroscópica. Histeroscopia en consulta. Minirresector. Bloqueo paracervical.

\section{Abstract}

Objective: To analyze the effectiveness of hysteroscopic myomectomy in office performed with mini-resectoscope, and to know if there is any variable related with patient satisfaction. Method: Observational and prospective transversal study, which included all women who underwent a hysteroscopic myomectomy in office in 2018. Patients received drugs for cervical preparation and pain management, as well as paracervical block. We used the $5.8 \mathrm{~mm}$ mini-resectoscope. We kept record of time and AVS pain during entrance and resection, as well as patient satisfaction 3 months after the procedure using the CSQ-8. Results: The study included 59 patients. Mean entrance time was less than 1 minute (47.93 seconds), while mean resection time was 13.51 minutes. AVS pain during entrance and resection was around 3 and 4, respectively. We achieved $74.6 \%$ rate of complete resection. Mean patient satisfaction rate was 27.17 points. We found that a complete myoma resection is related to higher patient satisfaction. Conclusions: Hysteroscopic myomectomy in office performed with the $5.8 \mathrm{~mm}$ mini-resectoscope, using cervical block, achieves good clinical results and a good patient satisfaction. Patient satisfaction is associated with a complete resection of the myoma, without any influence of pain experienced or time required.

Key words: Hysteroscopic myomectomy. Hysteroscopy in office. Mini-resectoscope. Cervical block.

Correspondencia:

*Rocío García-Jiménez

E-mail: rociogarji@gmail.com
Fecha de recepción: 10-11-2020

Fecha de aceptación: 08-07-2021

DOI: 10.24875/RECHOG.M21000015
Disponible en internet: $27-10-2021$ Rev Chil Obstet Ginecol. 2021;86(4):368-373

www. rechog.com 0048-766X / @ 2021 Sociedad Chilena de Obstetricia y Ginecología. Publicado por Permanyer. Éste es un artículo open access bajo la licencia CC BY-NC-ND (https://creativecommons.org/licenses/by-nc-nd/4.0/). 


\section{Introducción}

Los leiomiomas uterinos son los tumores pélvicos benignos más frecuentes en la mujer, con una prevalencia del $30-80 \%$ según la franja de edad y una incidencia máxima en torno a los 50 años. La mayoría de ellos son asintomáticos (50-80\%) y pueden pasar desapercibidos o ser un hallazgo incidental en una exploración ginecológica realizada por otro motivo. Cuando son sintomáticos (20-50\%), la clínica es muy diversa e incluye sangrado uterino anormal, síntomas compresivos derivados del efecto masa y síntomas reproductivos (como dificultad para la concepción o aumento de la tasa de aborto). A pesar de ser una patología benigna, el cuadro clínico puede llegar a generar un deterioro significativo de la calidad de vida de las pacientes, siendo hoy en día uno de los motivos de consulta más frecuentes en ginecología ${ }^{1,2}$.

En la actualidad, el desarrollo de las técnicas histeroscópicas ha supuesto un avance en el tratamiento de los miomas submucosos, ofreciendo una alternativa mínimamente invasiva segura y eficaz. Clásicamente estos procedimientos eran realizados bajo anestesia general o regional, pero gracias a la aparición de material de menor calibre ahora pueden realizarse en consulta, evitando así el riesgo anestésico, además de ofrecer un ahorro de tiempo y recursos económicos, y conseguir una mayor satisfacción de las pacientes ${ }^{3-5}$.

La realización de la histeroscopia en consulta está aceptada especialmente en el caso de las histeroscopias diagnósticas, y cada vez más autores abogan por seguir esta misma estrategia para las histeroscopias quirúrgicas, siguiendo el protocolo see and treat, sobre todo en el caso de los pólipos endometriales. En lo referente a los miomas submucosos, existen diferentes estrategias. Se tienen en cuenta determinados factores para clasificar la complejidad de extirpación de los miomas. La clasificación de Lasmar pronostica la dificultad de extirpación de los miomas según la puntuación de STEPW, la cual considera características del mioma como el tamaño, la topografía, la extensión de la base, la penetración en el miometrio y la afectación de la pared lateral. De esta forma se establecen tres categorías: I) miomectomía de baja complejidad; II) miomectomía de alta complejidad; y III) no abordable por histeroscopia ${ }^{6}$. En general, la histeroscopia en consulta se reserva para el abordaje de miomas de baja complejidad, utilizando material de pequeño calibre que limita la exéresis, mientras que las miomectomías de complejidad media-alta son abordadas en quirófano utilizando el resectoscopio ${ }^{7,8}$. Sin embargo, existen nuevas series de miomectomías de complejidad media realizadas en consulta, precisando completar la resección completa del mioma generalmente en más de una consulta ${ }^{9-11}$.

El objetivo principal de este estudio es analizar la efectividad de las miomectomías histeroscópicas en consulta realizadas con el minirresector en nuestro centro, considerando la efectividad como la resección completa del mioma. Como objetivo secundario, se busca conocer si existe relación entre los resultados de las miomectomías histeroscópicas en consulta y el grado de satisfacción de las pacientes.

\section{Método}

Se trata de un estudio observacional, transversal y prospectivo, llevado a cabo entre enero y diciembre de 2018. Todas las pacientes sometidas a miomectomía histeroscópica en consulta en nuestro centro fueron incluidas en el estudio, previa firma del consentimiento informado. Se recibió la aprobación del Comité de Ética del Hospital Universitario de Nuestra Señora de Valme, y se llevó a cabo siguiendo los principios éticos de la Declaración de Helsinki.

Las pacientes fueron sometidas a miomectomía histeroscópica en consulta según la práctica clínica habitual en nuestro centro. Todas recibieron medicación para la preparación cervical, que consistió en la administración de $200 \mu \mathrm{g}$ de misoprostol por vía oral 12 horas antes de la intervención. El día de la intervención, las pacientes tomaron con el desayuno $10 \mathrm{mg}$ de diazepam oral y $600 \mathrm{mg}$ de ibuprofeno oral, u otro analgésico oral en caso de contraindicación o reacción adversa medicamentosa conocida.

Para realizar la intervención, las pacientes recibieron anestesia paracervical. Tras la colocación del espéculo vaginal, visualizando el cuello uterino completo, pasamos a inyectar una ampolla de 1,8 $\mathrm{ml}$ de mepivacaína al $3 \%$ a nivel del fórnix posterior, en el límite entre la mucosa vaginal y cervical, en cada ligamento uterosacro, a las 5 y las 7 horarias. La infiltración de los ligamentos uterosacros con este anestésico pretende bloquear el dolor generado por la irritación del plexo presacro, que es el principal origen de la inervación sensitiva del útero. La mepivacaína tiene un inicio de acción rápido, con un periodo de latencia de 2-4 minutos y una duración intermedia de 1-4 horas.

Para realizar la miomectomía, el histeroscopio utilizado fue el minirresector Uteris $S$ bipolar de Palex (Invidia-Medical GmbH \& Co. KG, Tuttlingen, Germany), 
con un terminal de asa de un grosor de $5.8 \mathrm{~mm}(17.5$ Fr) y una óptica de $30^{\circ}$.

Durante la intervención se registraron el tiempo requerido para pasar a través del canal endocervical hasta lograr la entrada a la cavidad uterina y el tiempo empleado en la resección del mioma. Posteriormente, las pacientes indicaron el nivel de dolor percibido durante la entrada y la resección en una escala visual analógica (EVA).

Todas las pacientes fueron citadas en consulta de revisión a las 4-6 semanas. Se realizó una ecografía transvaginal para comprobar si la resección del mioma había sido completa.

Para conocer el grado de satisfacción de las pacientes, se contactó con ellas telefónicamente pasados 3 meses desde la intervención, y contestaron el cuestionario validado Client Satisfaction Questionnaire $(\mathrm{CSQ}-8)^{12}$. Se trata de un instrumento validado de evaluación breve con adecuadas propiedades psicométricas, utilizado de forma frecuente en ámbitos sanitarios. Consta de ocho preguntas en las que se evalúa de forma gradual de 1 a 4 el grado de satisfacción con el servicio recibido. La puntuación oscila entre 8 y 32 , estableciéndose tres niveles de satisfacción: bajo (8-20), medio (21-26) y alto (27-32) ${ }^{12,13}$.

De la historia clínica digitalizada se recogieron datos demográficos (edad), datos ginecológicos (paridad, cesáreas, partos vaginales, menopausia, tratamientos realizados) y datos referentes a las características del mioma (tamaño del mioma mayor, sintomatología, puntación STEPW, clasificación Lasmar y clasificación FIGO).

Las variables consideradas, en relación con los resultados de la histeroscopia, fueron el tiempo de entrada, el tiempo de resección, el dolor durante la entrada, el dolor durante la resección, el número de resecciones necesarias, la resección completa y la satisfacción total.

Los datos se analizaron con el paquete estadístico SPSS 20.0 para Windows. En primer lugar, respondiendo al primer objetivo del estudio para conocer los resultados obtenidos en nuestra unidad, se llevó a cabo un análisis descriptivo de los datos calculando valores de centralización y dispersión para las variables previamente descritas. A continuación, atendiendo al segundo objetivo, se tomaron las variables relacionadas con la técnica histeroscópica (tiempo de entrada, tiempo de resección, dolor durante la entrada, dolor durante la resección, resección completa) y se estudió la existencia de relación explicativa con la variable satisfacción total. Para ello se aplicó una regresión lineal múltiple.

\section{Resultados}

En el estudio se incluyeron 59 pacientes sometidas a miomectomías histeroscópicas. En primer lugar se analizaron las variables que definen la muestra (Tabla 1). La edad media de las pacientes fue de 46 años, siendo la mayoría de ellas multíparas (78\%) y en etapa premenopáusica (88.1\%). El $89.8 \%$ se encontraban sintomáticas y el tratamiento médico más utilizado fue el acetato de ulipristal (37.3\%). En cuanto a las características del mioma, el tamaño medio fue de $2.6 \mathrm{~cm}$, con una puntuación de STEPW media de 4.36, lo que supuso que la mayoría $(62.7 \%)$ se clasificaban como Lasmar I, que implica una resección fácil por histeroscopia.

Atendiendo al primer objetivo de nuestro estudio, se analizaron los resultados de la consulta (Tabla 2). El tiempo medio de entrada fue menor de 1 minuto (47.93 segundos) y el de resección fue de 13.51 minutos. El dolor referido por las pacientes en la EVA durante la entrada y la resección puntuó en torno a 3 y 4 , respectivamente. Se consiguió un $74.6 \%$ de resecciones completas de los miomas y la puntuación media de satisfacción de las pacientes fue de 27.17. Como se puede ver en la tabla 3, de las 15 resecciones incompletas, el $53.33 \%$ requirieron consecutivos procedimientos para completar la resección, y el otro $46.67 \%$ requirieron una histeroscopia en quirófano por dificultad técnica $(20 \%)$ o mala tolerancia al dolor $(6.67 \%)$, mientras que $3(20 \%)$ pacientes precisaron realización de histerectomía al ser la penetración intramural del mioma mayor del $50 \%$.

A continuación, procedimos a la realización del modelo de regresión múltiple para estudiar la asociación de la satisfacción total con el resto de las variables. Realizamos un análisis bivariante en el que solo resultó significativa la asociación con la resección completa del mioma $(p=0.021)$, como puede verse en la tabla 4. Para el modelo de regresión múltiple (Tabla 5) se incluyó dicha variable, así como el resto de las variables consideradas, ya que, a pesar de no haber mostrado significación estadística, su importancia clínica justifica su inclusión. El modelo resultó significativo ( $p=0.031$ ), con una R2 ajustada de 0.246. La resección total fue de nuevo la única variable que mostró significación estadística $(p=0.003)$ para ser incluida en el modelo, con un coeficiente de 3.618 y un intervalo de confianza del $95 \%$ de 1.264 a 5.972 . Así, el modelo ajustado final fue: satisfacción total $=24.391$ $+3.618 \cdot$ resección total. 
Tabla 1. Características de la muestra

\begin{tabular}{|c|c|}
\hline $\begin{array}{l}\text { Datos demográficos } \\
\text { Edad, media } \pm \mathrm{DE}\end{array}$ & $46 \pm 6.82$ \\
\hline $\begin{array}{l}\text { Datos ginecológicos } \\
\text { Partos vaginales, media } \pm \mathrm{DE} \\
\text { Cesáreas, media } \pm \mathrm{DE}\end{array}$ & $\begin{array}{l}1.47 \pm 1.13 \\
0.17 \pm 0.46\end{array}$ \\
\hline $\begin{array}{l}\text { Paridad, n (\%) } \\
\text { Nulíparas } \\
\text { Multíparas }\end{array}$ & $\begin{array}{l}13(22 \%) \\
46(78 \%)\end{array}$ \\
\hline $\begin{array}{l}\text { Menopausia, n (\%) } \\
\text { Premenopausia } \\
\text { Posmenopausia }\end{array}$ & $\begin{array}{c}52(88.1 \%) \\
7(11.9 \%)\end{array}$ \\
\hline $\begin{array}{l}\text { Tratamientos realizados, n }(\%) \\
\text { Progestágenos } \\
\text { Combinación estrógenos + progestágenos } \\
\text { Acetato de ulipristal }\end{array}$ & $\begin{array}{c}8(13.6 \%) \\
10(16.9 \%) \\
22(37.3 \%)\end{array}$ \\
\hline $\begin{array}{l}\text { Datos relacionados con los miomas } \\
\text { Sintomatología, } n(\%) \\
\text { Sintomáticas } \\
\text { Asintomáticas }\end{array}$ & $\begin{array}{c}53(89.8 \%) \\
6(10.2 \%)\end{array}$ \\
\hline Tamaño $(\mathrm{cm})$, media $\pm \mathrm{DE}$ & $2.6 \pm 1.14$ \\
\hline Puntuación STEPW, media \pm DE & $4.36 \pm 1.56$ \\
\hline $\begin{array}{l}\text { Clasificación Lasmar, n (\%) } \\
\text { Lasmar I } \\
\text { Lasmar II } \\
\text { Lasmar III }\end{array}$ & $\begin{array}{c}36(61 \%) \\
16(27.1 \%) \\
7(11.8 \%)\end{array}$ \\
\hline $\begin{array}{l}\text { Clasificación FIGO, n (\%) } \\
\text { FIGO } 0 \\
\text { FIGO I } \\
\text { FIGO II }\end{array}$ & $\begin{array}{c}14(23.7 \%) \\
37(62.7 \%) \\
8(13.6 \%)\end{array}$ \\
\hline
\end{tabular}

Tabla 2. Variables consideradas

\begin{tabular}{lc} 
Tiempo de entrada (segundos) & $47,93 \pm 32,75$ \\
\hline Tiempo de resección (minutos) & $13,51 \pm 11,38$ \\
\hline Puntuación EVA durante la entrada & $3,19 \pm 2,59$ \\
\hline Puntuación EVA durante la resección & $4,02 \pm 2,73$ \\
\hline Número de resecciones & $1,8 \pm 0,82$ \\
\hline Resección completa & \\
Si & $44(74,6 \%)$ \\
No & $15(25,4 \%)$ \\
\hline Puntuación CSQ-8 & $27,17 \pm 3,62$ \\
\hline
\end{tabular}

los datos se presentan en media \pm desviación estándar o frecuencias y porcentajes

De esta forma, encontramos que la resección completa del mioma se asocia con una mayor satisfacción de las pacientes, en comparación con la resección incompleta, aumentando 3.618 puntos más la
Tabla 3. Resecciones incompletas

\begin{tabular}{|l|c|}
\hline Resecciones incompletas & $15(100 \%)$ \\
\hline Requirieron consecutivas resecciones & $8(53.33 \%)$ \\
\hline Procedimiento en quirófano & $7(46.67 \%)$ \\
\hline $\begin{array}{l}\text { Dificultad técnica } \\
\text { Mala tolerancia al dolor }\end{array}$ & $3(20 \%)$ \\
\hline Histerectomía por penetrancia intramural > & $1(6.67 \%)$ \\
$50 \%$ & $3(20 \%)$ \\
\hline
\end{tabular}

Nota: los datos se presentan en frecuencias y porcentajes.

Tabla 4. Análisis bivariante

\begin{tabular}{|l|c}
\hline Variables & $p$ \\
\hline Tiempo de entrada (segundos) & 0.102 \\
\hline Tiempo de resección (minutos) & 0.25 \\
\hline Puntuación EVA durante la entrada & 0.513 \\
Puntuación EVA durante la resección & 0.697 \\
Resección completa & 0.021
\end{tabular}

Tabla 5. Modelo de regresión múltiple

\begin{tabular}{|l|c|c|}
\hline Variables & Coeficiente B (IC95\%) & p \\
\hline Tiempo de entrada & $-0.023(-0.054-0.008)$ & 0.14 \\
\hline $\begin{array}{l}\text { Tiempo de resección } \\
\begin{array}{l}\text { Puntuación EVA durante la } \\
\text { entrada }\end{array}\end{array}$ & $0.06(-0.029-0.149)$ & 0.179 \\
\hline $\begin{array}{l}\text { Puntuación EVA durante la } \\
\text { resección }\end{array}$ & $0.191(-0.245-0.628)$ & 0.381 \\
\hline \begin{tabular}{l} 
Resección completa \\
\hline
\end{tabular} & $3.618(1.264-5.972)$ & 0.003 \\
\hline
\end{tabular}

IC95\%: intervalo de confianza del 95\%.

puntuación, sin encontrar asociación estadísticamente significativa con el resto de las variables.

\section{Discusión}

A pesar de que la histeroscopia en consulta está cada vez más extendida y se recomienda siempre que sea posible, aún existen grupos que presentan reticencias al respecto, en especial en el caso de las histeroscopias quirúrgicas ${ }^{14}$. En nuestro centro seguimos la estrategia see and treat en consulta, en la que en un mismo acto se realiza la evaluación de la cavidad uterina y se procede al tratamiento quirúrgico de los hallazgos patológicos.

El motivo principal para no realizar miomectomías histeroscópicas en consulta es la intolerancia al dolor 
de las pacientes, al precisar histeroscopios de mayor diámetro que necesitan más dilatación cervical, por lo que se recomienda material de menos de $5 \mathrm{~mm}$ o flexible para las histeroscopias en consulta ${ }^{15}$. En nuestro centro utilizamos el minirresector de $5.8 \mathrm{~mm}$, obteniendo buenos resultados, con una tasa de resección completa en un único acto quirúrgico de casi el $75 \%$. Al utilizar material de un diámetro algo mayor que el recomendado es necesaria una correcta preparación cervical, motivo por el cual se administran sistemáticamente $200 \mu \mathrm{g}$ de misoprostol por vía oral 12 horas antes, dado que ha demostrado ser la estrategia que obtiene mejores resultados ${ }^{16-19}$.

Uno de los aspectos que más preocupa y limita el uso de la histeroscopia en consulta es el manejo del dolor durante el procedimiento. Según la literatura, para las histeroscopias quirúrgicas más sencillas se recomienda el uso de bloqueo paracervical en los uterosacros por su bajo coste, buena tolerancia y reducción del dolor, mientras que las miomectomías histeroscópicas generalmente requieren analgesia adicional además del bloqueo paracervical, como sedación intravenosa 0 incluso general 0 raquídea ${ }^{15,20,21}$. Sin embargo, nuestro estudio muestra unas puntuaciones moderadas en la EVA, entre 3 y 4 , consiguiendo una resección completa del mioma en casi el $75 \%$ de las ocasiones, con un tiempo de realización menor de 15 minutos de media. Por lo tanto, según nuestros resultados, la miomectomía histeroscópica en consulta es una opción apropiada con buenos resultados en la mayoría de las pacientes.

En cuanto a las complicaciones, la literatura reporta una tasa del $0.22 \%$ para los procedimientos histeroscópicos, siendo la más frecuente la perforación uterina, con un $0.12 \%^{22}$. Por tanto, no es de extrañar que no se hayan producido complicaciones en los procedimientos incluidos en nuestro estudio.

Dado que los miomas submucosos son un problema relativamente frecuente en ginecología, el hecho de que sea factible extirparlos en la consulta promueve beneficios en cuanto a costes y racionalización de los recursos. La histeroscopia en consulta tiene beneficios directos para la paciente al evitar el riesgo anestésico y la hospitalización, y permitir una temprana incorporación a su actividad diaria. Además, al tratarse de un acto que tiene lugar en una única consulta, las pacientes pueden continuar con su actividad normal, sin necesitar ingreso hospitalario.

Si bien los beneficios desde el punto de vista médico son numerosos, también es necesario conocer la opinión de las pacientes con el fin de observar si estos beneficios se reflejan en la satisfacción de ellas. Observamos en nuestro estudio que la satisfacción total de las pacientes fue buena, con una puntuación media de 27 puntos sobre un total de 32 en el CSQ-8. Cabe mencionar, sin embargo, que no se ha estudiado la relación de la satisfacción de las pacientes con los diferentes factores demográficos, ginecológicos o del mioma, considerando esto como una de las limitaciones del estudio.

Se encontró que el factor que estadísticamente se asociaba a una mayor satisfacción de la paciente no era el dolor, ni el tiempo necesario para la resección del mioma, sino la resección completa del mioma. Es decir, las pacientes más satisfechas son aquellas en las que se logró la extirpación completa del mioma, sin importar si ello implicaba un mayor o menor tiempo, o dolor. Esto podría deberse al hecho de que, una vez resecado el mioma, las pacientes no tienen que volver a la consulta, y dada la desaparición de los síntomas, el problema se soluciona y mejora su calidad de vida. Esto nos hacer pensar que, si bien es importante reducir los tiempos de resección por los efectos indeseados que su prolongación pudiese tener, además de un correcto manejo del dolor, el factor que más valoran las pacientes es la resección completa, y los demás aspectos quedan relegados a un segundo plano.

\section{Conclusiones}

Hemos conseguido un número significativo de miomectomías histeroscopias efectivas en consulta con anestesia paracervical usando el minirresector de $5.8 \mathrm{~mm}$, con una media de dolor de leve a moderado y una alta satisfacción las pacientes. Esta última se relaciona con una resección completa del mioma, sin que influyan el tiempo necesario para su exéresis ni el dolor. En definitiva, la miomectomía histeroscópica en consulta con analgesia paracervical es una opción adecuada que obtiene buenos resultados clínicos y gran satisfacción de las pacientes.

\section{Financiamiento}

El presente trabajo se llevó a cabo sin financiamiento.

\section{Conflicto de intereses}

Los autores declaran no tener conflictos de intereses. 


\section{Responsabilidades éticas}

Protección de personas y animales. Los autores declaran que los procedimientos seguidos se conformaron a las normas éticas del comité de experimentación humana responsable y de acuerdo con la Asociación Médica Mundial y la Declaración de Helsinki.

Confidencialidad de los datos. Los autores declaran que han seguido los protocolos de su centro de trabajo sobre la publicación de datos de pacientes.

Derecho a la privacidad y consentimiento informado. Los autores han obtenido el consentimiento informado de los pacientes y/o sujetos referidos en el artículo. Este documento obra en poder del autor de correspondencia.

\section{Bibliografía}

1. Day Baird D, Dunson DB, Hill MC, Cousins D, Schectman JM. High cumulative incidence of uterine leiomyoma in black and white women: ultrasound evidence. Am J Obstet Gynecol. 2003;188:100-7.

2. Okolo S. Incidence, aetiology and epidemiology of uterine fibroids. Best Pract Res Clin Obstet Gynaecol. 2008:22:571-88.

3. Marana R, Marana E, Catalano GF. Current practical application of office endoscopy. Curr Opin Obstet Gynecol. 2001;13:383-7.

4. Fothergill RE. Endometrial ablation in the office setting. Obstet Gynecol Clin North Am. 2008;35:317-30.

5. Kremer C, Duffy S, Moroney M. Patient satisfaction with outpatient hysteroscopy versus day case hysteroscopy: randomised controlled trial. BMJ. 2000;29:279-82.

6. Lasmar RB, Lasmar BP, Celeste RK, da Rosa DB, Depes D de B Lopes RG. A new system to classify submucous myomas: a Brazilian multicenter study. J Minim Invasive Gynecol. 2012;19:575-80.
7. Moawad NS, Santamaria E, Johnson M, Shuster J. Cost-effectiveness of office hysteroscopy for abnormal uterine bleeding. JSLS. 2014;18:e2014.00393.

8. Dealberti D, Riboni F, Cosma S, Pisani C, Montella F, Saitta S, et al. Feasibility and acceptability of office-based polypectomy with a 16F mini-resectoscope: a multicenter clinical study. J Minim Invasive Gynecol. 2016;23:418-24.

9. Ma T, Readman E, Hicks L, Porter J, Cameron M, Ellett L, et al. Is outpatient hysteroscopy the new gold standard? Results from an 11 year prospective observational Study. Aust N Z J Obstet Gynaecol. 2017:57:74-80.

10. Salazar CA, Isaacson KB. Office operative hysteroscopy: an update. J Minim Invasive Gynecol. 2017;25:199-208.

11. Ahmad G, Attarbashi S, O'Flynn H, Watson AJ. Pain relief in office gynecology: a systematic review and meta-analysis. Eur J Obstet Gynecol Reprod Biol. 2011;155:3-13.

12. Larsen DL, Attkisson CC, Hargreaves WA, Nguyen TD. Assessment of client/patient satisfaction: development of a general scale. Eval Program Plann. 1979;2:197-207.

13. Vázquez FL, Torres A, Otero P, Blanco V, Attkisson CC. Propiedades psicométricas de la versión castellano-castellano del Cuestionario de Satisfacción del Cliente (CSQ-8). Psicología Actual. 2019;38:829-35.

14. Giorda G, Scarabelli C, Franceschi S, Campagnutta E. Feasibility and pain control in outpatient hysteroscopy in postmenopausal women: a randomized trial. Acta Obstet Gynecol Scand. 2000;79:593-7.

15. Readman E, Maher PJ. Pain relief and outpatient hysteroscopy: a literature review. J Am Assoc Gynecol Laparosc. 2004;11:315-9.

16. Al-Fozan H, Firwana B, Al Kadri H, Hassan S, Tulandi T. Preoperative ripening of the cervix before operative hysteroscopy. Cochrane Database Syst Rev 2015;(4):CD005998.

17. Oppegaard KS, Lieng M, Berg A, Istre O, Qvigstad E, Nesheim BI. A combination of misoprostol and estradiol for preoperative cervical ripening in postmenopausal women: a randomised controlled trial. BJOG. 2010;117:53-61.

18. Ngai SW, Chan YM, Ho PC. The use of misoprostol prior to hysteroscopy in postmenopausal women. Hum Reprod. 2001;16:1486-8.

19. Fung TM, Lam MH, Wong SF, Ho LC. A randomised placebo-controlled trial of vaginal misoprostol for cervical priming before hysteroscopy in postmenopausal women. BJOG. 2002;109:561-5.

20. Tangsiriwatthana T, Sangkomkamhang US, Lumbiganon $P$ Laopaiboon M. Paracervical local anaesthesia for cervical dilatation and uterine intervention. Cochrane Database Syst Rev. 2013;(9):CD005056.

21. Bettocchi S, Ceci O, Di Venere R, Pansini MV, Pellegrino A, Marello F, et al. Advanced operative office hysteroscopy without anaesthesia: analysis of 501 cases treated with a 5 Fr. bipolar electrode. Hum Reprod. 2002;17:2435-8.

22. Aydeniz B, Gruber IV, Schauf B, Kurek R, Meyer A, Wallwiener D. A multicenter survey of complications associated with 21,676 operative hysteroscopies. Eur J Obstet Gynecol Reprod Biol. 2002;104:160-4. 